\title{
Vacancy-mediated mechanism of nitrogen substitution in carbon nanotubes
}

\author{
Deepak Srivastava* \\ NASA Ames Research Center, CSC, Mail Stop T27-A1, Moffett Field, California 94035-1000, USA \\ Madhu Menon ${ }^{\dagger}$ \\ Department of Physics and Astronomy, University of Kentucky, Lexington, Kentucky 40506-0055, USA \\ and Center for Computational Sciences, University of Kentucky, Lexington, Kentucky 40506-0045, USA \\ C. Daraio and S. Jin \\ Department of Mechanical and Aerospace Engineering, University of California, San Diego, La Jolla, California 92093-0411, USA \\ Bindu Sadanadan and Apparao M. Rao \\ Department of Physics and Astronomy, Clemson University, Clemson, South Carolina 29634, USA
}

(Received 18 February 2004; published 30 April 2004)

\begin{abstract}
Nitrogen substitution reaction in a graphene sheet and carbon nanotubes of different diameter are investigated using the generalized tight-binding molecular dynamics method. The formation of a vacancy in curved graphene sheet or a carbon nanotube is found to cause a curvature dependent local reconstruction of the surface. Our simulations and analysis show that vacancy mediated $\mathrm{N}$ substitution (rather than $\mathrm{N}$ chemisorption) is favored on the surface of nanotubes with diameter larger than $8 \mathrm{~nm}$. This predicted value of the critical minimum diameter for $\mathrm{N}$ incorporation is confirmed by experimental results presented on nitrogen-doped multiwalled nanotubes with $\approx 5$ at. $\%$ nitrogen prepared by the thermal chemical vapor deposition process.
\end{abstract}

DOI: 10.1103/PhysRevB.69.153414

PACS number(s): 61.48.+c, 71.15.Pd, 81.10.Bk

The discovery of carbon nanotubes (CNTs) by Iijima, ${ }^{1}$ has set off intensive studies of these quasi-one-dimensional structures. The single-wall nanotubes consist of rolled-up single graphene sheets of carbon atoms into nanotubes of different diameters and chiralities. Due to their chirality dependent electrical properties, and excellent mechanical and thermal properties, CNTs have been suggested for applications in molecular electronics, functional composite materials, thermal management on a chip, and gas or drug storage and delivery devices. ${ }^{2,3}$ The possibility of the existence of nanotubes made of noncarbon elements such as boron-nitride (BN), B- and N-doped carbon nanotubes have also received significant attention, ${ }^{4-16}$ where successful synthesis involve arc discharge methods, ${ }^{8}$ reduction of carbon nanotube via substitution reactions, ${ }^{10-12}$ and thermal (chemical vapor deposition) CVD. ${ }^{16}$

Significance of substitution reaction in a carbon nanotube by $\mathrm{B}, \mathrm{N}$ and other species is considerable from both materials and electronics perspective. Multiwall N-doped CNTs have been synthesized in large quantities with the morphology of a bamboo-shoot-like structure, ${ }^{16}$ and B and or N doping of $\mathrm{C}$ nanotube can be used to engineer the band gaps of these quasi-one-dimensional materials for electronics applications. ${ }^{17}$ Moreover, specifically substitutional doping of carbon nanotubes with $\mathrm{B}$ and $\mathrm{N}$ elements has also been reported at high-temperature annealing experiments. ${ }^{11,12} \mathrm{By}$ definition, a substitution reaction of a $\mathrm{N}$ atom in a CNT involves replacement of $\mathrm{C}$ atom in the structure of the CNT with an adsorbing $\mathrm{N}$ atom in the surroundings. Therefore, conceptually a mechanism for substitution reaction with in a nanotube may involve a three step process: (a) creation of a vacancy or a defect in an otherwise perfect structure of the $\mathrm{CNT}$, and (b) incorporation of the external substituting atom (like $\mathrm{N}$ in this case) at the newly created vacancy or defect site, and (c) equilibration or redistribution, via diffusion, of one or many substituted atoms into energetically favorable morphologies or configurations in the systems. Activation barriers involved in any or all of these three contributing steps may be high but much of the synthesis processes are performed at high temperatures and extreme conditions in which diffusion or creation of vacancies may be very facile.

In this Brief Report, we investigate a vacancy mediated mechanism of $\mathrm{N}$ doping of $\mathrm{C}$ nanotubes through, (a) energetics of vacancy formation in the CNT wall as a function of nanotube diameter or curvature of the nanotube surface, and (b) incorporation of $\mathrm{N}$ at the vacancy sites, through the generalized tight-binding molecular dynamics (GTBMD) scheme of Menon and Subbaswamy. ${ }^{18}$ The theoretical calculations are complemented by experimental measurements for the same systems. For small diameter (or high-curvature) CNT surfaces, the formation of a vacancy causes a reconstruction at the vacancy site, whereas in large diameter nanotubes with low surface curvature dangling bonds are stable. We find that $\mathrm{N}$ incorporation as a function of surface curvature is of chemisorption type in the former and of substitution type in the latter. A critical lower limit of the diameter of CNTs for $\mathrm{N}$ substitution is predicted and verified by experimental observation of diameter distribution of the $\mathrm{N}$-doped CNTs synthesized using thermal CVD.

The theoretical methods used in the present work is the GTBMD scheme of Menon and Subbaswamy, ${ }^{18}$ which allows for dynamic relaxation of the system with no symmetry constraints. Successful applications of the GTBMD method to C (Ref. 18), Si (Ref. 19), BN (Refs. 15 and 20) and $\mathrm{SiC}^{21}$ systems have been reported elsewhere. The GTBMD method 
has been used to predict that zigzag $\mathrm{BN}$ nanotubes are more stable and, therefore, should be more readily made in experiments. ${ }^{15}$ Recent experimental findings have provided a striking confirmation of this prediction by finding that, regardless of the experimental conditions used in their production, zigzag arrangement always dominates $\mathrm{BN}$ nanotubes. ${ }^{12}$ In the present work we use the GTBMD method to obtain structural and energetic characterization of vacancy formation and $\mathrm{N}$ incorporation in CNTs as a function of nanotube surface curvature. Our experimental method for preparing $\mathrm{N}$-doped CNTs is based on a CVD method which produces high quality multiwall carbon nanotubes (MWCNTs) in bulk quantities. ${ }^{22}$ In this method the average tube diameter ranges between $25-30 \mathrm{~nm}$, with a noticeable fraction of tubes with diameter below $10 \mathrm{~nm}^{23}$

The energetics of vacancy creation and $\mathrm{N}$ incorporation in CNTs were done by computing the total energy differences using the GTBMD method on (i) a bare graphene sheet, (ii) graphene sheet with a vacancy and relaxation induced reconstruction, and (iii) a $\mathrm{N}$ atom incorporated at the site of the vacancy. A full set of the energetics as a function of curvature was then obtained by repeating the above sequence and curving the graphene sheet with curvature corresponding to the range of external diameter of N-doped MWCNTs observed in experiments. This allows us to do many energetics calculations over a range of curvatures corresponding to diameters varying from $1 \mathrm{~nm}$ to $80 \mathrm{~nm}$ to a completely flat graphene sheet. The sheet is chosen large enough so that the simulated region is sufficiently far from the edge carbon atoms. For a given curvature the edge $\mathrm{C}$ atoms are held fixed and all other atoms are dynamically allowed to relax to a minimum-energy configuration.

The strain energy as a function of surface curvature for a graphene sheet with and without a vacancy is shown in Fig. 1(a). The energies are given relative to the corresponding flat graphene sheet with and without the vacancy, respectively. In the absence of a vacancy the curve represents the strain energy due to the surface curvature alone (also a measure of nanotube formation energy in a different context) and increases as the surface curvature increases (or as the tube diameter decreases) from that of a flat graphene sheet. As expected, the increase in the strain energy is monotonic for the defect free CNTs of smaller diameter. Note that the strain energy for the defect free CNT follows a $1 / d^{2}$ behavior. The results were computed for tube diameters as large as $80 \mathrm{~nm}$, but only shown for up to $40 \mathrm{~nm}$ because the changes in the energies for tube diameters larger than $30 \mathrm{~nm}$ are negligible at the scale of energetics shown in Fig.1(a).

The change in the strain energy as a function of diameter for the graphene sheet containing vacancy, however, is not monotonic and shows an interesting low-energy feature for small diameters (large curvature) CNTs as seen in Fig.1(a). Note that the two smooth curves shown in the figure are drawn over many computed points used in the interpolation. For clarity in discussion we have shown the positions of only points $A, B$, and $C$ along the potential well curve for the graphene sheet containing vacancy. These points correspond to diameters $2 \mathrm{~nm}, 5 \mathrm{~nm}$, and $8 \mathrm{~nm}$, respectively. As the figure shows, the strain energy remains fairly constant be-
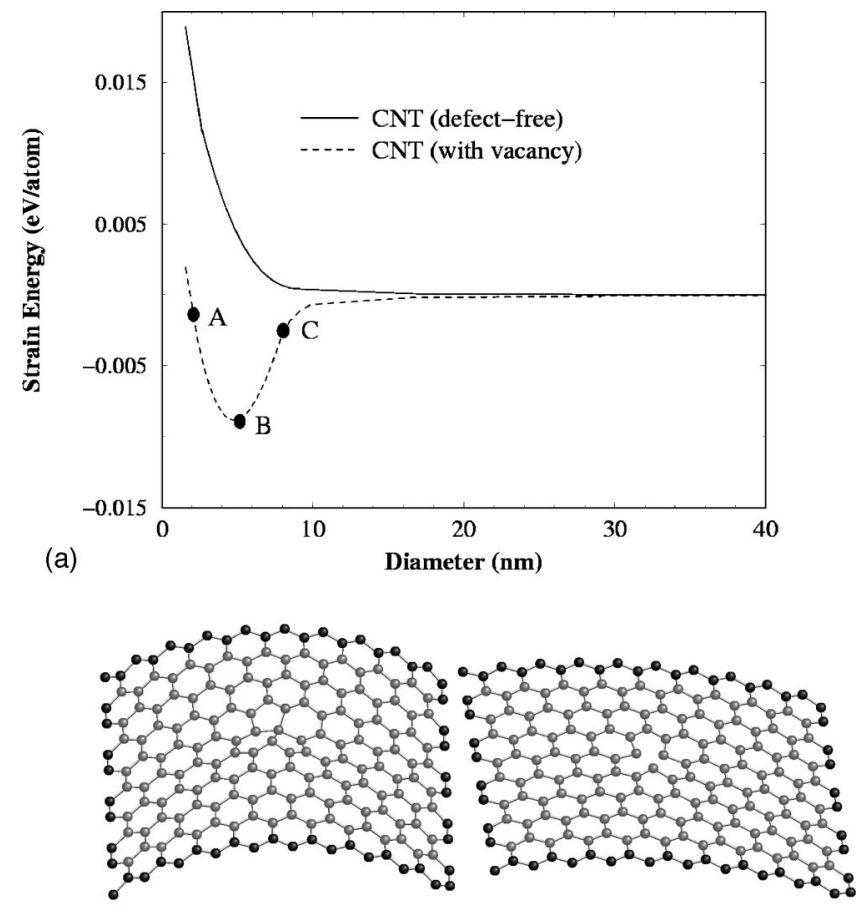

(b)

FIG. 1. (a) Strain energy of a curved graphene sheet with and without a vacancy as a function of curvature expressed as corresponding nanotube diameter in nanometers. (b) The left and right panels show the surface reconstructions formed at the vacancy site for nanotube diameters corresponding to points $\mathrm{B}$ and $\mathrm{C}$, respectively. The edge carbon atoms held fixed during the simulations are shown in dark.

yond point $C$. Our simulations show the vacancy induced reconstructions to undergo a qualitative change while crossing the point $C$ corresponding to a nanotube diameter of 8 $\mathrm{nm}$ indicating the existence of a critical diameter. The reconstruction for diameters less than $8 \mathrm{~nm}$ is shown on the left panel of Fig. 1(b) where the reconstruction corresponding to point $B$ is shown. As seen in the figure, a $\mathrm{C}$ atom moves towards the location of the vacancy and forms two pentagons sharing a vertex. The central $\mathrm{C}$ atom in this case has four strained $\mathrm{C}-\mathrm{C}$ bonds. The reconstruction for diameters greater than $8 \mathrm{~nm}$ is shown on the right panel of Fig. 1(b) where the reconstruction corresponding to point $C$ is shown. Here the $\mathrm{C}$ atoms surrounding the vacancy do not move towards the location of the vacancy. All three $\mathrm{C}$ atoms around the location of the vacancy remain equivalent and one can conceptually visualize this reconstruction as a structure formed by three incomplete pentagons with possibly six strained C-C bonds (two each for the three carbon atoms surrounding the vacancy) in the lattice. This reconstruction remain stable for larger diameter nanotubes. The structure shown on the right panel of Fig. 1(b) (for large diameter nanotubes, corresponding to point $C$ ) is, thus, energetically less stable than the structure shown on the left panel of Fig. 1(b) (corresponding to point $B$ ) due to different bonding reconstruction. Furthermore, even though bonding reconstructions at $A$ and $B$ are the same, from Fig. 1(a), it can be seen that the structure corresponding to point $A$ is energetically less stable than the 


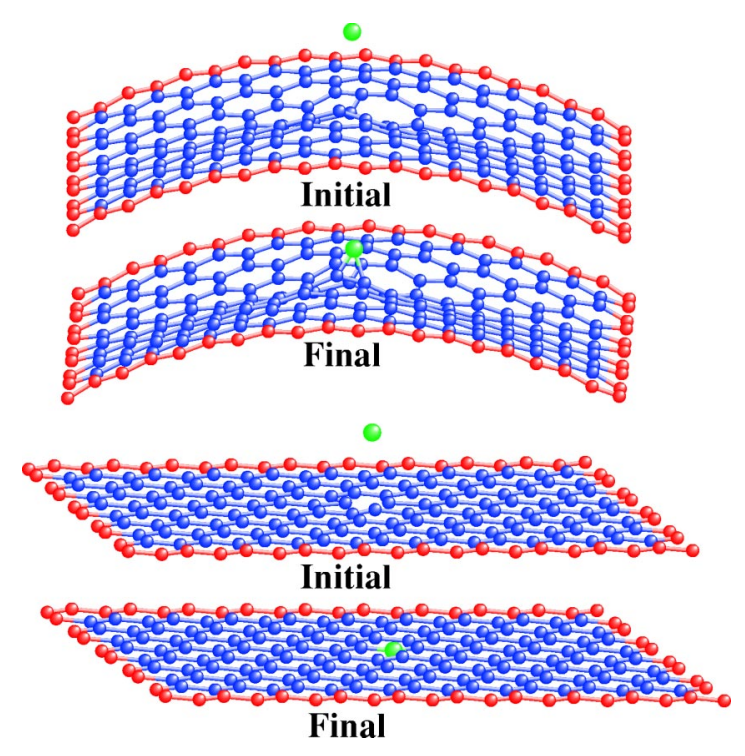

FIG. 2. (Color online) (a) Chemisorption-type $\mathrm{N}$ incorporation at reconstruction site corresponding to point B in Fig. 1(a), and (b) $\mathrm{N}$ substitution at the reconstruction site corresponding to a point beyond $\mathrm{C}$ in Fig. 1(a), representing a large diameter tube.

structure at $B$ due to the larger curvature induced strain at $A$. An interesting feature of the reconstruction shown on the left panel of Fig. 1(b) is that due to the pulling in of $\mathrm{C}$ atom at the shared vertex of the two pentagons, the structure is asymmetrically nonplanar. The reconstruction shown on the right panel of Fig. 1(b), on the other hand, keeps the structure symmetrically nonplanar throughout. This suggests that such a reconstruction is stable on surfaces corresponding to small curvature or large diameter $(>8 \mathrm{~nm})$ nanotubes.

The approach and incorporation of a $\mathrm{N}$ atom on the two types of reconstructions are shown in Figs. 2(a) and 2(b). A number of trajectories were used in our simulations of $\mathrm{N}$ atom approaching the two types of reconstructions. We find that for off-center approaches, $\mathrm{N}$ atom on the reconstruction shown on the left panel of Fig. 1(b) has a tendency to form a chemisorbed-type bond with the lattice. On the reconstruc- tion shown on the right panel of Fig. 1(b), in all cases, the N atom gets incorporated in the lattice as in a substitution reaction. The surface curvature and off-planar nature of the reaction in the first case is visible in Fig. 2(a), while in Fig. 2(b) the structure before and after the reaction and incorporation remains planar. The above simulations and analysis show that vacancy mediated $\mathrm{N}$ incorporation with substitution type morphology will be favored for surfaces of CNTs of diameter of about $8 \mathrm{~nm}$ or larger. For smaller diameters CNTs, initial chemisorption is favored which could be followed by, (a) diffusion of the N atom on the CNT surface, (b) reverse reaction and also sometimes, (c) incorporation of the $\mathrm{N}$ atom in the lattice with the substitution morphology.

The method for producing bulk quantities of high-purity aligned MWCNT films through the catalytic decomposition of ferrocene-xylene mixture at a temperature of $700{ }^{\circ} \mathrm{C}$ has been discussed in detail elsewhere. ${ }^{22}$ Briefly, a feed solution of ferrocene-xylene $(0.75-1.0$ at. \% Fe) is injected into a two-stage tubular quartz reactor using a syringe pump. Highpurity aligned MWCNT films were produced in bulk quantities on bare quartz substrates placed inside the reactor. For producing films of nitrogen-doped nanotubes, melamine was introduced simultaneously $\left(\mathrm{C}_{3} \mathrm{H}_{6} \mathrm{~N}_{6}\right.$, Aldrich Chemicals, $\mathrm{T}_{\text {sublimation }}=300^{\circ} \mathrm{C}$ ) during the growth of MWCNT films. A quartz boat containing $3 \mathrm{~g}$ of melamine was placed inside the quartz tube reactor in a region between the preheater and the furnace. ${ }^{22}$ The location of the quartz boat was carefully chosen to ensure no sublimation of melamine as the preheater and furnace attain their operating temperatures of 200 and $700{ }^{\circ} \mathrm{C}$, respectively. After the preheater and the furnace temperatures were stabilized, the syringe pump was turned on to inject the ferrocene-xylene mixtures, and the entire quartz tube was pushed towards the exhaust side so that the boat now resides in a region of the furnace where the temperature is sufficiently high to sublime melamine. The ferrocene-xylene injection rate was maintained at $1 \mathrm{ml} / \mathrm{hr}$, and upon entering the preheater, the ferrocene-xylene mixture was immediately volatized and swept into the furnace by a flow of argon $(675 \mathrm{sccm})$ and hydrogen $(75 \mathrm{sccm})$. Typical

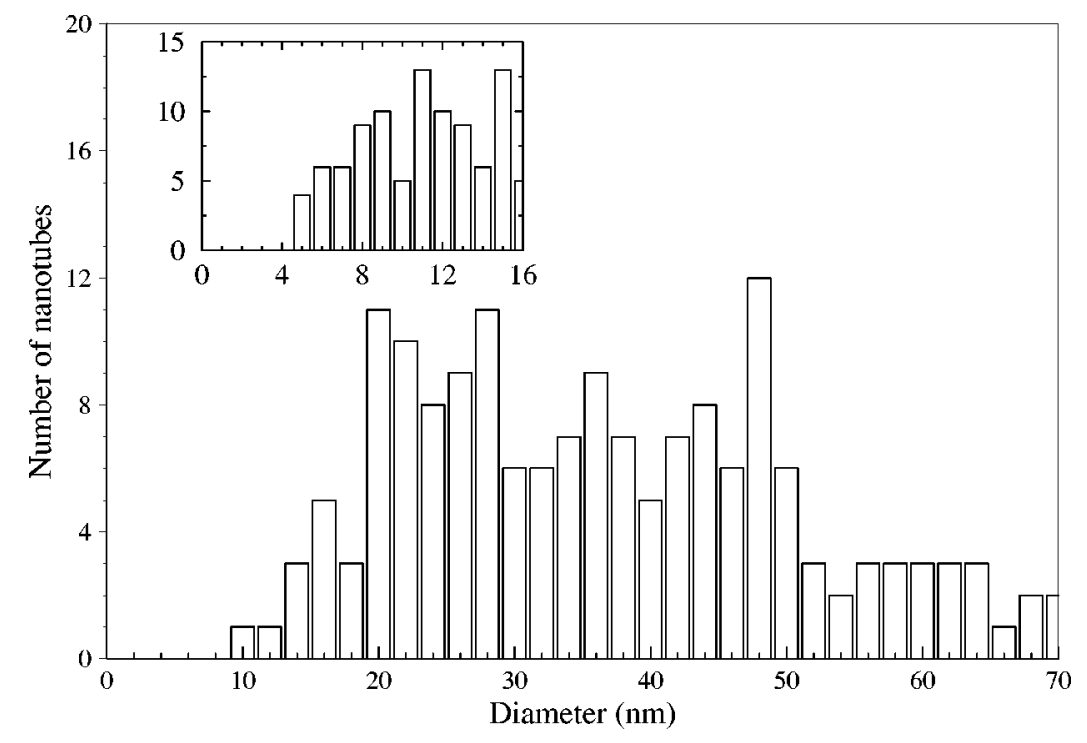

FIG. 3. Diameter distribution of N-doped MWCNTs produced by thermal CVD experiment described in the text. Diameter distribution of the undoped sample is shown in the inset. The inset clearly indicates that MWCNTs with diameter $<10 \mathrm{~nm}$ are present in the samples. 
run time was 15 min during which time all melamine sublimed. After the reaction, the preheater and the furnace were allowed to cool to room temperature in flowing argon. Carbon deposits formed on the walls of the quartz tube and substrates were extracted and characterized using transmission electron microscopy (TEM). For measuring the diameter of N-doped nanotubes, a cluster of nanotube deposit was dispersed ultrasonically in tetrahydrofuran (THF). A drop of the suspension was placed on a TEM grid and subsequently analyzed. From the TEM images the diameter of the nanotubes was measured and number of nanotubes as a function of diameter is shown in Fig. 3. Electron energy loss spectroscopy revealed that $\approx 5$ at. $\%$ nitrogen was present in the nanotubes and extensive characterization of the nitrogendoped tubes used in this study can be found in Ref. 24. From the plot it is evident that majority of $\mathrm{N}$-doped nanotubes $(>70 \%)$ are in the range of $20-50 \mathrm{~nm}$ in diameter. Significantly, we do not observe any N-doped CNT in the sample where the nanotube diameter is about $10 \mathrm{~nm}$ or smaller. The diameter distribution of the undoped sample is shown in the inset which clearly indicates that MWNTs with diameter $<10 \mathrm{~nm}$ are present in the samples. Identical synthesis conditions were used to prepare nitrogen-doped tubes. However, in the case of nitrogen-doped tubes, we do not find tubes with diameter less than $10 \mathrm{~nm}$. This observation supports the above described theoretical prediction where vacancy medi- ated substitution reaction of dopant species through pyridinelike reconstruction is not favored for smaller diameter single or multiwall carbon nanotubes. It is interesting to note that several reports on B substitution or B combined with $\mathrm{N}$ substitution in SWNT bundles can be found in the literature while no hard evidence for $\mathrm{N}$ substitution in SWNTs has been reported. Furthermore, consistent with findings of this study, several studies on N substitution in isolated SWNTs has been reported. ${ }^{16,17}$

In summary, a vacancy mediated substitution of $\mathrm{N}$ in carbon nanotubes as a function of tube diameter has been investigated and described in this work. We find that, as a function of tube diameter, the formation of a pyridinelike vacancy defect and the substitution of the dopant species ( $\mathrm{N}$ in our case) at the defect site is favored over chemisorption for tubes of diameters larger than about $8 \mathrm{~nm}$. In the smaller diameter nanotubes $(<8 \mathrm{~nm})$, chemisorption or formation of fused pentagon-type defects is favored.

D.S. is supported by NASA Contract No. DTTS59-99-D00437 to CSC. M.M. gratefully acknowledges grant support from NSF (Grant No. ITR-0221916), U.S. DOE (Grant No. 00-63857), NASA (Grant No. 02-65679), and KSTC (Grant No. 03-66986). A.M.R. gratefully acknowledges support under grants through NASA Ames Research Center and NSF (ERC EEC-9731680 and NIRT 0304019 and 0244290).
*Electronic address: deepak@nas.nasa.gov

†Electronic address: super250@pop.uky.edu

†Electronic address: arao@CLEMSON.EDU

${ }^{1}$ S. Iijima, Nature (London) 354, 56 (1991).

${ }^{2}$ D. Srivastava, M. Menon, and K. Cho, Comput. Sci. Eng. 3, 42 (2001).

${ }^{3}$ M. Meyyappan and D. Srivastava, in CRC Handbook of Nanoscience, Engineering and Technology, edited by W.A. Goddard, D.W. Brenner, S.E. Lyshevski, and G.J. Iafrate (CRC Press, Boca Raton, FL, 2002), Vol. 18, p. 1.

${ }^{4}$ A. Rubio, J.L. Corkill, and M.L. Cohen, Phys. Rev. B 49, 5081 (1994).

${ }^{5}$ X. Blase, A. Rubio, S.G. Louie, and M.L. Cohen, Europhys. Lett. 28, 335 (1994).

${ }^{6}$ P. Gleize, M.C. Schouler, P. Gadelle, and M. Caillet, J. Mater. Sci. 29, 1575 (1994).

${ }^{7}$ P. Gleize, S. Herreyre, P. Gadelle, M. Mermoux, M.C. Cheynet, and L. Abello, J. Mater. Sci. Lett. 13, 1413 (1994).

${ }^{8}$ N.G. Chopra, R.J. Luyken, K. Cherry, V.H. Crespi, M.L. Cohen, S.G. Louie, and A. Zettl, Science 269, 966 (1995).

${ }^{9}$ A. Loiseau, F. Willaime, N. Demoncy, G. Hug, and H. Pascard, Phys. Rev. Lett. 76, 4737 (1996).

${ }^{10}$ D. Goldberg, Y. Bando, W. Han, K. Kurashima, and T. Sato, Chem. Phys. Lett. 308, 337 (1999).
${ }^{11}$ D. Goldberg, W. Han, Y. Bando, L.B.K. Kurashima, and T. Sato, J. Appl. Phys. 86, 2364 (1999).

${ }^{12}$ D. Goldberg, Y. Bando, K. Kurashima, and T. Sato, Solid State Commun. 116, 1 (2000).

${ }^{13}$ X. Blase, A.D. Vita, J.C. Charlier, and R. Car, Phys. Rev. Lett. 80, 1666 (1998).

${ }^{14}$ E. Hernandez, C. Goze, P. Bernier, and A. Rubio, Phys. Rev. Lett. 80, 4502 (1998).

${ }^{15}$ M. Menon and D. Srivastava, Chem. Phys. Lett. 307, 407 (1999).

${ }^{16}$ M. Terrones and H. Terrones, Appl. Phys. Lett. 75, 3932 (1999).

${ }^{17}$ R. Czerw et al., Nano Lett. 1, 457 (2001).

${ }^{18}$ M. Menon, E. Richter, and K.R. Subbaswamy, J. Chem. Phys. 104, 5875 (1996).

${ }^{19}$ M. Menon and K. Subbaswamy, Phys. Rev. B 55, 9231 (1997).

${ }^{20}$ D. Srivastava, M. Menon, and K. Cho, Phys. Rev. B 63, 195413 (2001).

${ }^{21}$ M. Menon, J. Chem. Phys. 114, 7731 (2001).

${ }^{22}$ R. Andrews, D. Jacques, A.M. Rao, F. Derbyshire, D. Qian, X. Fan, and E.C. Dickey, Chem. Phys. Lett. 303, 467 (1999).

${ }^{23}$ S.B. Sinnott, R. Andrews, D. Qian, A.M. Rao, Z. Mao, E.C. Dickey, and F. Darbyshire, Chem. Phys. Lett. 315, 25 (1999).

${ }^{24}$ B. Sadanadan, T. Savage, S. Bhattacharya, T. Tritt, A. Cassell, M. Meyyappan, Z.R. Dai, R.Z.Z.L. Wang, and A.M. Rao, J. Nanosci. Nanotechnol. 3, 99 (2003). 